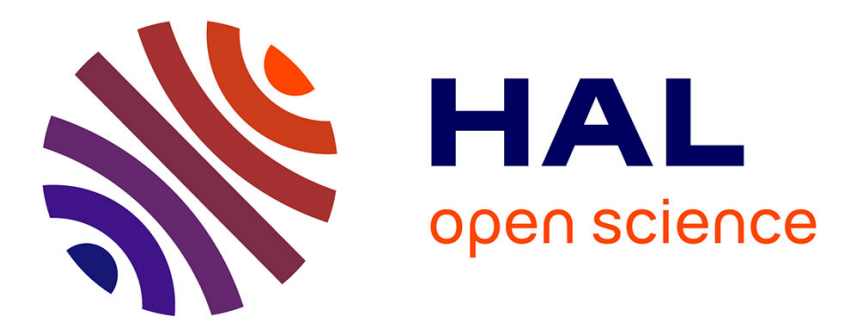

\title{
Sponsored search engines in competition: advertisers behavior and engines optimal ranking strategies
}

\author{
Patrick Maillé, Bruno Tuffin
}

\section{To cite this version:}

Patrick Maillé, Bruno Tuffin. Sponsored search engines in competition: advertisers behavior and engines optimal ranking strategies. 19th IEEE International Symposium on Modelling, Analysis and Simulation of Computer and Telecommunication Systems (MASCOTS 2011), Jul 2011, Singapore, Singapore. pp.96-103, 10.1109/MASCOTS.2011.19 . hal-00640709

\section{HAL Id: hal-00640709 \\ https://hal.science/hal-00640709}

Submitted on 14 Nov 2011

HAL is a multi-disciplinary open access archive for the deposit and dissemination of scientific research documents, whether they are published or not. The documents may come from teaching and research institutions in France or abroad, or from public or private research centers.
L'archive ouverte pluridisciplinaire HAL, est destinée au dépôt et à la diffusion de documents scientifiques de niveau recherche, publiés ou non, émanant des établissements d'enseignement et de recherche français ou étrangers, des laboratoires publics ou privés. 


\section{Sponsored Search Engines in Competition: Advertisers Behavior and Engines Optimal Ranking Strategies}

\author{
Patrick Maillé \\ Institut Telecom; Telecom Bretagne \\ 2, rue de la Châtaigneraie CS 17607 \\ 35576 Cesson Sévigné Cedex, France \\ Email: patrick.maille@telecom-bretagne.eu
}

\author{
Bruno Tuffin \\ INRIA Rennes - Bretagne Atlantique \\ Campus Universitaire de Beaulieu \\ 35042 Rennes Cedex, France \\ Email: Bruno.Tuffin@inria.fr
}

\begin{abstract}
Search engines are essential actors for web browsing. We analyze here the economic competition between search engines earning money from adword auctions. We develop a twolevel game where at the largest time scale search engines decide which allocation rule to implement, between revenue-based and bid-based; and at the lowest time-scale advertisers decide how to split their advertising budget between the two search engines, depending on the benefits this will bring to them. The game at the largest time scale is solved using backward induction, the search engines anticipating the reactions of advertisers. We describe the advertisers best strategies and show how to determine, depending on parameters, an equilibrium on the ranking rule strategy for search engines; this may explain Yahoo!'s move to switch from bid-based to revenue-based ranking to follow Google's strategy.
\end{abstract}

\section{INTRODUCTION}

When using current telecommunication networks, and more specifically the Internet, reaching a desired content is in most cases obtained through a search engine, unless the user knows exactly the targeted web address. Those search engines, such as Google, Yahoo! or Bing, propose a ranked list of web sites corresponding to the keywords that have been typeset. But, being commercial entities, they make money by additionally presenting advertisement links, usually at the top and/or on the right of the page. The advertisement links are selected from an auction to which an advertiser can submit a bid if she wants to be displayed, because she believes the keyword search may result in a financial transaction when the user sees the ad. Search engine advertising has become an important business, the combined revenue of the two main actors in the area, Yahoo! and Google, counting for more than $\$ 11$ billion in 2005 [1], this business being also expected to count for about $40 \%$ of total advertising revenue [2].

There are many ways to define the list of displayed ads (the allocation rule), and when and how much the advertisers will pay the search engine (the pricing rule). Typically, there are $K$ available slots for each keyword search. Advertisers may pay the search engine each time the ad is displayed (Pay-Per-Impression model), each time the ad is clicked (PayPer-Click model), or each time the click results in a sell (Pay-Per-Transaction model). In most if not all cases, the
Pay-Per-Click model is implemented by search engines. The bids can be ranked, and allocated, either according to the bids (the so-called bid-based ranking), or according to the revenue they can generate when Pay-Per-Click is chosen, by considering the product of the bid and the Click-Through-Rate (CTR), which is the proportion of time the ad is clicked when displayed (giving the so-called revenue-based ranking). About the amount charged to each advertiser, there exist also several possible rules: first-price auctions directly charge the submitted bid, while Vickrey-Clarke-Groves (VCG) auctions (see [3], [4], among others) charge the loss in terms of total (sum of) utilities of all others actors that the advertiser's presence imposes on others. VCG is considered in many economic contexts since it is known to satisfy relevant properties such as the incentive for advertisers to submit their true valuation for the service of being displayed. But the Generalized Second Price (GSP) auction is actually the scheme considered by search engines. It consists in charging you the bid value that would make your ranking value equal to the one just below you. For bid-based ranking, you pay the bid below you; for revenue-based ranking, you pay the amount that makes the revenue generated from you equal to the revenue obtained from the one below you if she were charged her bid. You therefore always pay less than your bid. GSP is explained to be used instead of VCG mechanism because it brings to the engine a higher revenue [5]. This paragraph briefly explained how adword auctions work; for more information, the reader is advised to look at [5], [6], [7], [8].

While there is an extensive literature on the analysis of sponsored search auctions, investigating the best strategies of advertisers and search engines, it has to be remarked that there are surprisingly very few papers dealing with search engines in competition for advertisers [2], [9], [10], [11]. Though, the optimization of strategies can hardly be realized without taking care of that competition. Our goal in this paper is to study the competition between search engines in economic competition, as well as the advertisers' best strategies in this context. We consider two engines, each one offering a single commercial slot, and having a fixed (not necessarily equal) part 
of the search market. Both engines apply the GSP charging scheme, as commonly admitted now, but they have to decide which ranking strategy to implement, between bid-based and revenue-based. Their decisions depend on the strategy of the competitor (hence the use of non-cooperative game theory [12]), but also on the reaction of advertisers who have a fixed budget per unit of time and need to decide how to split their advertising budget on the engines. Note that the decisions of advertisers depend on the reaction of other advertisers and the ranking strategies of engines. We therefore end up with a twolevel game, solved by backward induction: the game between engines, although played first, is analyzed by anticipating what the reactions of advertisers would be.

The existing papers on competitive sponsored search auctions can be described and compared with ours as follows. The goal in [2] is to determine the best auction rule for engines offering a single slot and to understand the impact of ranking policies in a competitive environment, considering the different possible pairs of ranking policies employed by the two search engines (bid-based or revenue-based). It is assumed that advertisers pertain to two classes and submit an ad only once, i.e., that have to choose between the two engines, and Nash equilibria in mixed strategies are obtained. Our model does not consider this last assumption about advertisers submitting bids only once, but rather considers the more realistic situation of two advertisers with a fixed budget and splitting this budget between engines to optimize their interest. Competition is also considered in [9], where a double auction with advertisers on one side and slot sellers on the other is analyzed; but the goal of the model is only to study the efficiency and truthful-bidding properties when the assumption of CTR separability (the CTR being the product of a positiondependent term, independent of advertiser, and an advertiserrelated term, independent of the position) is satisfied or not. Otherwise, [10] analyzes the competition between two engines applying VCG auctions, each engine offering a potentially different number of slots, and having different CTRs. The advertisers' game is solved depending on the fact that they can or cannot submit bids at both engines. Our model is closer to reality, assuming that GSP is applied, and investigating the search engines best strategy. We could also cite [11], but the model does not specifically address sponsored search auctions and GSP mechanism for instance. Remark that bid-based and revenue-based rankings have been compared in [13], but in the simpler case of a monopoly; no game was then involved in the model.

The paper is organized as follows. Section II presents the model and its basic assumptions on the search engines and advertisers, with two search engines proposing a single slot and two advertisers having a budget that they wish to split optimally among engines. Section III presents and computes the key performance measures of the model, the average price charged and the winning probability, expressed in terms of the considered advertiser's valuation. They are computed for both the cases of bid-based and revenue-based rankings. Section IV then analyzes the game between two advertisers in competition for the advertising slots, making use of the previously computed performance measures. On top of that advertisers game, Section V explains how engines can strategically decide which allocation rule to implement, between bid-based and revenuebased, in order to maximize their revenue. Finally, Section VI concludes by summarizing the main findings of the paper and presenting directions for future research.

\section{MOdEL}

Before analyzing the two-level game, we present here the model assumptions about the search engines and advertisers behavior.

\section{A. Search engines}

We assume that we have two search engines (SE), labelled 1 and 2 in competition for advertisers. Each engine proposes a single advertisement slot. We consider a single keyword, and fix to $\lambda$ the average number of searches per unit of time for that keyword.

We also define as $\alpha$ (with $0 \leq \alpha \leq 1$ ) the (fixed) market share of SE 1, meaning that $\alpha \lambda$ searches are performed on SE 1 while $(1-\alpha) \lambda$ are performed on SE 2 . The underlying assumption is that the search engine selection does not depend on the ads, but rather on the engine presentation, reputation and choice of regular (organic) links; this seems reasonnable to us.

Both search engines are assumed to apply GSP charging scheme, where the ranking of advertisers can be bid-based or revenue-based.

\section{B. Advertisers}

We assume for the general model presentation that there are $k$ advertisers in competition for the advertising slots. Each advertiser is characterized by her budget $b$, taken from cdf $G(b)$ and her valuation per click, taken from the cdf $F(v)$. All budgets and valuations are independent and independent between advertisers.

The CTR of an advertiser at an SE is assumed to be separable, as the product of the CTR of the search engine, $q_{1}$ and $q_{2}$ for SE 1 and 2 respectively, and of the CTR $c_{i}$ of the considered Advertiser $i$. This is a usual assumption of most adword auctions models [8].

The goal of Advertiser $i$, with budget $b_{i}$ and valuation $v_{i}$, is to distribute her budget $b_{i}$ among the two search engines. Since SEs apply GSP mechanism on a single slot (GSP is then equivalent to VCG), we can expect advertisers to be truthful, that is to submit a bid equal to her valuation, which will always result in a benefit. As a consequence, advertisers are better off using all their budget. We therefore define $\beta_{i}$ as the proportion of budget submitted to SE 1 , while $1-\beta_{i}$ is submitted to SE 2. It is the parameter Advertiser $i$ can play with.

Let $w_{j}\left(v_{i}\right)$ denote the probability that $i$ wins on $\mathrm{SE} j$ given that her bid (valuation) is $v_{i}$. The average gain of Avertiser $i$ when its link is clicked on engine $j$, is $v_{i}-\mathbb{E}\left[R_{j} \mid v_{i}\right], \mathbb{E}\left[R_{j} \mid v\right]$ being the average price paid on SE $j$ given that the advertiser has won the auction when bidding $v$, the revenue $R_{j}$ of SE $j$ 
being random, dependent on competitors' valuations, budgets, and choices. The rates at which Advertiser $i$ submits her bids to SE 1 and SE 2 are then respectively

$$
\begin{aligned}
& \lambda_{i}^{(1)}=\min \left(\frac{\beta_{i} b_{i}}{q_{1} c_{i} \mathbb{E}\left[R_{1} \mid v_{i}\right] w_{1}\left(v_{i}\right)}, \alpha \lambda\right) \\
& \lambda_{i}^{(2)}=\min \left(\frac{\left(1-\beta_{i}\right) b_{i}}{q_{2} c_{i} \mathbb{E}\left[R_{2} \mid v_{i}\right] w_{2}\left(v_{i}\right)},(1-\alpha) \lambda\right) .
\end{aligned}
$$

To better explain the first term in the minimum, it represents the number of times Advertiser $i$ can bid in order to reach her budget repartition, in average: the average budget is indeed the money paid at each click times the probability of a click times the probability to be displayed. Note that we consider that the objective so far is not to spend the whole budget but rather to approach the target budget repartition. In particular, when it is not possible to spend $\beta_{i} b_{i}$ on SE 1 while the budget constraint $\left(1-\beta_{i}\right) b_{i}$ is limiting, then the remaining budget is not affected to SE 2. Nevertheless, when studying the advertisers best $\beta_{i}$ such situations will not occur since reducing the $\beta_{i}$ would result in a better outcome for advertiser $i$.

If we define $p_{i}^{(j)}$ as the probability that $i$ bids at $\operatorname{SE} j$, we get, from $p_{i}^{(1)}=\lambda_{i}^{(1)} /(\alpha \lambda)$ and $p_{i}^{(2)}=\lambda_{i}^{(2)} /((1-\alpha) \lambda)$,

$$
\begin{aligned}
& p_{i}^{(1)}=\min \left(\frac{\beta_{i} b}{\alpha \lambda q_{1} c_{i} \mathbb{E}\left[R_{1} \mid v_{i}\right] w_{1}\left(v_{i}\right)}, 1\right) \\
& p_{i}^{(2)}=\min \left(\frac{\left(1-\beta_{i}\right) b}{(1-\alpha) \lambda q_{2} c_{i} \mathbb{E}\left[R_{2} \mid v_{i}\right] w_{2}\left(v_{i}\right)}, 1\right) .
\end{aligned}
$$

Let $\beta=\left(\beta_{1}, \ldots, \beta_{k}\right)$ the vector/profile of strategies of advertisers. The utility of $i, U_{i}(\beta)$ represents the revenue made by $i$ per unit of time from the sales due to clicks. We have

$$
\begin{aligned}
U_{i}(\beta)= & q_{1} c_{i} w_{1}\left(v_{i}\right) \lambda_{i}^{(1)}\left(v_{i}-\mathbb{E}\left[R_{1} \mid v_{i}\right]\right) \\
& +q_{2} c_{i} w_{2}\left(v_{i}\right) \lambda_{i}^{(2)}\left(v_{i}-\mathbb{E}\left[R_{2} \mid v_{i}\right]\right) .
\end{aligned}
$$

Remark that, according to Equations (1) and (2), deciding $\beta_{i}$ for Advertiser $i$ is equivalent to determining the probability $p_{i}$ to submit a bid. An important assumption here is that advertisers know their own bid, their only other knowledge of other players is assumed to be the distribution of valuations and budgets and the observation of their bidding probability. Indeed, we do not consider that advertisers try to deduce the valuation and budget of competitors from their bidding probabilities.

We thus have a game between advertisers to determine how to optimally split their budget. In order to analyze this game, and afterwards the ranking game between search engines, we need to compute the average price and winning probability at an SE, which of course depend on the allocation and pricing mechanism applied.

\section{AVERAGE PRICES AND WINNING PROBABILITIES AT SEARCH ENGINES}

To simplify the notations, we just consider in this section a single SE, and that the budget of Advertiser $i$ is $b_{i}$ (it will be replaced by $\beta_{i} b_{i}$ and $\left(1-\beta_{i}\right) b_{i}$ when dealing with the competitive case). We also define $\lambda_{i}$ as the rate at which $i$ will compete, out of the total rate of $\lambda$ (which will be $\alpha \lambda$ or $(1-\alpha) \lambda$ when split between SEs). The bidding probability of $i$ is then $p_{i}=\lambda_{i} / \lambda$. We compute the average prices and winning probabilities at search engines when bid-based or revenuebased rankings are applied.

\section{A. With bid-based ranking}

We first assume that bid-based ranking is applied. When facing no other bidder, the expected price is 0 and the probability of winning is 1 . When facing opponents, the probability $w(v)$ of winning with bid/valuation $v$ is the probability that all other participants bid less than $v$, but considering also all possible combinations of bidders. Let $V_{\ell}$ be the random valuation of Advertiser $\ell$. Define also $j_{\ell}=1$ if $\ell$ participates to the auction, and $j_{\ell}=0$ otherwise. We denote by $\pi_{\ell}=p_{\ell} j_{\ell}+\left(1-p_{\ell}\right)\left(1-j_{\ell}\right)$ the probability of the bidding action of $\ell$. The probability to win with valuation $v$ is the probability that among all other bidders (among the $k-1$ competitors), their valuation is less than $v$ :

$$
\begin{aligned}
w(v) & =\sum_{j_{1}, \ldots, j_{k-1} \in\{0,1\}} \mathbb{P}\left[\forall j_{\ell}=1, V_{\ell}<v\right]\left(\prod_{\ell=1}^{k-1} \pi_{\ell}\right) \\
& =\sum_{j_{1}, \ldots, j_{k-1} \in\{0,1\}}(F(v))^{\left(\sum_{l=1}^{k-1} j_{l}\right)}\left(\prod_{\ell=1}^{k-1} \pi_{\ell}\right) .
\end{aligned}
$$

That winning probability will be the one $a$ priori expected by an advertiser with valuation $v$. Again, we use the assumption that advertisers do not try to use the participation of the competitors to estimate their valuations.

The expected price the advertiser will have to pay is the expected value of the largest bid among all other bids given that they all bid less than $v$. The density function of bidders knowing that they bid less than $v$ is $h(u)=f(u) / F(v)$ with cdf $H(u)=F(u) / F(v)$ for $u \leq v$. The density of the largest bid among the $j$ other bidders is then

$$
\frac{j(F(u))^{j-1} f(u)}{(F(v))^{j}} .
$$

The expected price $\mathbb{E}[R \mid v]$ is the expected value of the second largest bid, taking into account the probabilities that each bidder submits a bid. We end-up with:

$$
\begin{aligned}
\mathbb{E}[R \mid v]= & \sum_{j_{1}, \ldots, j_{k-1} \in\{0,1\}}\left(\prod_{\ell=1}^{k-1} \pi_{\ell}\right) \\
& \int_{u \leq v} \frac{\left(\sum_{l=1}^{k-1} j_{l}\right) u(F(u))^{\left(\sum_{l=1}^{k-1} j_{l}\right)-1} f(u)}{(F(v))^{\left(\sum_{l=1}^{k-1} j_{l}\right)}} d u .
\end{aligned}
$$

We therefore get a system of equations to determine the $w_{j}\left(v_{i}\right), \mathbb{E}\left[R_{j}\right]$ and $p_{i}^{(j)}$, since the $p_{i}^{(j)}$ depend on the $w_{j}\left(v_{i}\right)$ and $\mathbb{E}\left[R_{j}\right]$, while the $w_{j}\left(v_{i}\right)$ and $\mathbb{E}\left[R_{j}\right]$ themselves depend on the $p_{i}^{(j)}$.

In the case of symmetric advertisers, assuming that all advertisers have the same valuation and budget, but are only 
aware of the distribution of competitors, we obtain

$$
\begin{aligned}
w(v) & =\sum_{\ell=0}^{k-1}\left(\begin{array}{c}
k-1 \\
\ell
\end{array}\right)(F(v))^{\ell}\left(p_{1}\right)^{\ell}\left(1-p_{1}\right)^{k-1-\ell} \\
& =\left(F(v) p_{1}+1-p_{1}\right)^{k-1} .
\end{aligned}
$$

The expected price is:

$$
\begin{aligned}
\mathbb{E}[R \mid v]= & \sum_{\ell=0}^{k-1}\left(\begin{array}{c}
k-1 \\
\ell
\end{array}\right)\left(p_{1}\right)^{\ell}\left(1-p_{1}\right)^{k-1-\ell} \\
& \int_{u \leq v} \frac{\ell u(F(u))^{\ell-1} f(u)}{(F(v))^{\ell}} d u \\
= & \sum_{\ell=0}^{k-1}\left(\begin{array}{c}
k-1 \\
\ell
\end{array}\right)\left(p_{1}\right)^{\ell}\left(1-p_{1}\right)^{k-1-\ell} \\
& \left(v-\int_{u \leq v}\left(\frac{F(u))}{F(v))}\right)^{\ell} d u\right) .
\end{aligned}
$$

Along with

$$
p_{1}=\min \left(\frac{b_{1}}{\lambda q c_{1} \mathbb{E}[R \mid v] w(v)}, 1\right),
$$

this gives us a set of three equations with three unknowns. Replacing in (6) $\mathbb{E}[R \mid v]$ and $w(v)$ by their expressions in terms of $p_{1}$, we get an equation in $p_{1}$ that can easily be solved numerically.

Example 1: Assuming that $F$ is a uniform distribution on $[0, a]$, the above equation in $p_{1}$ becomes

$p_{1}=\min (1$,

$\left.\frac{b_{1}}{\lambda q c_{1} v\left(\sum_{\ell=0}^{k-1}\left(\begin{array}{c}k-1 \\ \ell\end{array}\right) p_{1}^{\ell}\left(1-p_{1}\right)^{k-1-\ell} \frac{\ell}{\ell+1}\right)\left(\frac{v}{a} p_{1}+1-p_{1}\right)^{k-1}}\right)$

When $k=2$, if there is a solution in $p_{1}$ with $0<p_{1}<1$, it is a solution of the polynom of degree 3 :

$$
p_{1}^{2} \lambda q c_{1} v \frac{1}{2}\left(\frac{v}{a} p_{1}+1-p_{1}\right)=b_{1} .
$$

With $\lambda=1000, a=10, b=40, q=0.5, c_{1}=0.5$ and $v=5$, this polynom has a single solution, $p_{1}=0.27218$. On the other hand, it may happen that there is no solution in $(0,1)$ (for instance if $\lambda=100, a=10, b=100, q=0.5, c_{1}=0.5$ and $v=5$ ). When it is the case, it means that, necessarily, $p_{1}=1$ because the value in the minimum is always larger than 1. Indeed, if there is no $p_{1}$ such that $\frac{b}{\lambda q c_{1} \mathbb{E}[R \mid v] w(v)}<1$, it is also the case when $p_{1}=1$, leading to this last value and the corresponding $w(v)$ and $\mathbb{E}[R \mid v]$. When we have more than one solution, the advertisers can choose between several strategies, and will choose the one providing the larger utility.

We have therefore shown that there always exists a solution in the case $k=2$ and a uniform valuation distribution. This is straightforward to generalize, to give the following proposition.
Proposition 1: In the symmetric case, there always exists a solution to the system of equations providing the values of $p_{1}, w(v)$ and $\mathbb{E}[R \mid v]$.

Proof: The right-hand expression of (6) can be seen to be continuous in $p_{1} \in[0,1]$ since $\mathbb{E}[R \mid v]$ is nonnegative and continuous in $p_{1}$. Since a solution of the system of equations is a fixed-point of that expression, the existence of such a solution is ensured by Brouwer's fixed point theorem.

Consider now the case of two advertisers only, but such that the SE imposes a reserve price $p_{r}$ in case there is only one bidding advertiser. We end up with a system of six equations with six unknowns (with $v_{1}, v_{2} \geq p_{r}$ to avoid degenerate cases):

$$
\begin{aligned}
p_{1}= & \min \left(\frac{b_{1}}{\lambda q c_{1} \mathbb{E}\left[R_{1} \mid v_{1}\right] w\left(v_{1}\right)}, 1\right) \\
p_{2}= & \min \left(\frac{b_{2}}{\lambda q c_{2} \mathbb{E}\left[R_{2} \mid v_{2}\right] w\left(v_{2}\right)}, 1\right) \\
w\left(v_{1}\right)= & \left(1-p_{2}\right)+F\left(v_{1}\right) p_{2} \\
w\left(v_{2}\right)= & \left(1-p_{1}\right)+F\left(v_{2}\right) p_{1} \\
\mathbb{E}\left[R_{1} \mid v_{1}\right]= & \left(1-p_{2}\right) p_{r} \\
& +p_{2}\left(p_{r} \frac{F\left(p_{r}\right)}{F\left(v_{1}\right)}+\int_{p_{r} \leq u \leq v_{1}} \frac{u f(u)}{F\left(v_{1}\right)} d u\right) \\
\mathbb{E}\left[R_{2} \mid v_{2}\right]= & \left(1-p_{1}\right) p_{r} \\
& +p_{1}\left(p_{r} \frac{F\left(p_{r}\right)}{F\left(v_{2}\right)}+\int_{p_{r} \leq u \leq v_{2}} \frac{u f(u)}{F\left(v_{2}\right)} d u\right) .
\end{aligned}
$$

Here again, the system can be solved numerically, by looking at the four cases $\left(p_{1}<1\right.$ and $\left.p_{2}<1\right),\left(p_{1}=1\right.$ and $\left.p_{2}<1\right)$, $\left(p_{1}<1\right.$ and $\left.p_{2}=1\right)$, and $\left(p_{1}=1\right.$ and $\left.p_{2}=1\right)$.

Example 2: Considering again $F$ uniform over $[0, a]$, we get

$$
\begin{aligned}
p_{1} & =\min \left(\frac{b_{1}}{\lambda q c_{1} \mathbb{E}\left[R_{1} \mid v_{1}\right] w\left(v_{1}\right)}, 1\right) \\
p_{2} & =\min \left(\frac{b_{2}}{\lambda q c_{2} \mathbb{E}\left[R_{2} \mid v_{2}\right] w\left(v_{2}\right)}, 1\right) \\
w\left(v_{1}\right) & =\left(1-p_{2}\right)+\frac{v_{1}}{a} p_{2} \\
w\left(v_{2}\right) & =\left(1-p_{1}\right)+\frac{v_{2}}{a} p_{2} \\
\mathbb{E}\left[R_{1} \mid v_{1}\right] & =\left(1-p_{2}\right) p_{r}+p_{2}\left(\frac{\left(p_{r}\right)^{2}}{2 v_{1}}+\frac{v_{1}}{2}\right) \\
\mathbb{E}\left[R_{2} \mid v_{2}\right] & =\left(1-p_{1}\right) p_{r}+p_{1}\left(\frac{\left(p_{r}\right)^{2}}{2 v_{2}}+\frac{v_{2}}{2}\right) .
\end{aligned}
$$

If $p_{1}<1$ and $p_{2}<1$, we have a system of two equations in $\left(p_{1}, p_{2}\right)$ :

$$
\begin{aligned}
& p_{1}=\frac{b_{1}}{\lambda q c_{1}\left[\left(1-p_{2}\right) p_{r}+p_{2}\left(\frac{\left(p_{r}\right)^{2}}{2 v_{1}}+\frac{v_{1}}{2}\right)\right]\left(1-p_{2}+\frac{v_{1}}{a} p_{2}\right)} \\
& p_{2}=\frac{b_{2}}{\lambda q c_{2}\left[\left(1-p_{1}\right) p_{r}+p_{1}\left(\frac{\left(p_{r}\right)^{2}}{2 v_{2}}+\frac{v_{2}}{2}\right)\right]\left(1-p_{1}+\frac{v_{2}}{a} p_{1}\right)},
\end{aligned}
$$

giving an equation in $p_{1}$ which can be solved numerically. The cases $\left(p_{1}=1\right.$ and $\left.p_{2}<1\right),\left(p_{1}<1\right.$ and $\left.p_{2}=1\right)$, and $\left(p_{1}=1\right.$ and $\left.p_{2}=1\right)$ can be processed similarly. For instance, 
if $a=20, \lambda=100, q=0.5, b_{1}=10, c_{1}=0.5, b_{2}=40$, $c_{2}=0.4, v_{1}=8, v_{2}=5$ and $p_{r}=0.5$, no solution exists with $0<p_{1}, p_{2}<1$ or $p_{1}=1, p_{2}<1$ or $p_{1}=p_{2}=1$. But we get a single solution $p_{2}=1$ and $p_{1}=0.2490272374$.

\section{B. With revenue-based ranking}

We assume that all the $c_{\ell}$ are known by competitors, deduced from observations. If $V_{\ell}$ denotes again the random valuation of $\ell$, following the same idea than in (4), an advertiser with valuation $v$ and CTR $q c$ will win an auction in front of $k-1$ competitors if her product $q c v$ is larger than the $q c_{\ell} V_{\ell}$ of each other bidding advertiser $\ell$. This occurs with probability

$$
\begin{aligned}
w(v) & =\sum_{j_{1}, \ldots, j_{k-1} \in\{0,1\}} \mathbb{P}\left[\forall j_{\ell}=1, c_{\ell} V_{\ell}<c v\right]\left(\prod_{\ell=1}^{k-1} \pi_{\ell}\right) \\
& =\sum_{j_{1}, \ldots, j_{k-1} \in\{0,1\}} \prod_{\ell=1}^{k-1}\left(F\left(v \frac{c}{c_{\ell}}\right)\right)^{j_{\ell}} \pi_{\ell} .
\end{aligned}
$$

Recall that for an opponent $\ell$ with CTR $q c_{\ell}$, the cumulative distribution function (cdf) of the revenue is $\mathbb{P}\left[q c_{\ell} V \leq x\right]=$ $F\left(x /\left(q c_{\ell}\right)\right)$. To determine the expected price given that the considered advertiser with valuation $v$ and CTR $q c$ wins, we need the density of the highest bid among opponents, conditionnally to providing a product CTR times valuations less than $c q v$. The revenues of competitors are then in $[0, v q c]$. The conditional cdf for $\ell$ is

$$
\frac{\mathbb{P}\left[q c_{\ell} V \leq x, q c_{\ell} V \leq q c v\right]}{\mathbb{P}\left[q c_{\ell} V \leq q c v\right]}=\frac{F\left(\min \left(x /\left(q c_{\ell}\right), v c / c_{\ell}\right)\right)}{F\left(v c / c_{\ell}\right)},
$$

from which we can then deduce the average price per click (dividing the revenue by the CTR):

$$
\begin{aligned}
\mathbb{E}[R \mid v]=\frac{1}{c q} \sum_{j_{1}, \ldots, j_{k-1} \in\{0,1\} \mid}\left(\prod_{\ell=1}^{k-1} \pi_{\ell}\right) & \\
& \int_{x \leq v q c} \frac{\sum_{\ell: j_{\ell}=1} x \frac{1}{q c_{\ell}} \prod_{l=1 ; l \neq \ell}^{k-1}\left(F\left(x /\left(q c_{l}\right)\right)\right)^{j_{l}} f\left(x /\left(q c_{\ell}\right)\right)}{\prod_{\ell=1}^{k-1}\left(F\left(v \frac{c}{c_{\ell}}\right)\right)^{j_{\ell}}} d x .
\end{aligned}
$$

Remark that in the case of symmetric advertisers (with the same CTR $c$ ), the revenue-based ranking becomes exactly the bid-based ranking, and the results of the previous subsection follow.

In the case of two advertisers only, assuming here too that the SE imposes a reserve revenue $r_{r}$ in case there is only one bidding advertiser or the bid of the opponent leads to less average revenue than $r_{r}$, we end up with a system of six equations with six unknowns:

$$
\begin{aligned}
p_{1}= & \min \left(\frac{b_{1}}{\lambda q c_{1} \mathbb{E}\left[R_{1} \mid v_{1}\right] w\left(v_{1}\right)}, 1\right) \\
p_{2}= & \min \left(\frac{b_{2}}{\lambda q c_{2} \mathbb{E}\left[R_{2} \mid v_{2}\right] w\left(v_{2}\right)}, 1\right) \\
w\left(v_{1}\right)= & \left(1-p_{2}\right)+F\left(v_{1} c_{1} / c_{2}\right) p_{2} \\
w\left(v_{2}\right)= & \left(1-p_{1}\right)+F\left(v_{2} c_{2} / c_{1}\right) p_{1} \\
q c_{1} \mathbb{E}\left[R_{1} \mid v_{1}\right]= & \left(1-p_{2}\right) r_{r}+p_{2}\left(r_{r} \frac{F\left(r_{r} /\left(q c_{2}\right)\right)}{F\left(v_{1} c_{1} / c_{2}\right)}\right. \\
& \left.+\int_{r_{r} \leq x \leq v_{1} q c_{1}} \frac{\left(1 /\left(q c_{2}\right)\right) x f\left(x /\left(q c_{2}\right)\right)}{F\left(v_{1} c_{1} / c_{2}\right)} d x\right) \\
q c_{2} \mathbb{E}\left[R_{2} \mid v_{2}\right]= & \left(1-p_{1}\right) r_{r}+p_{1}\left(r_{r} \frac{F\left(r_{r} /\left(q c_{1}\right)\right)}{F\left(v_{2} c_{2} / c_{1}\right)}\right. \\
& \left.+\int_{r_{r} \leq x \leq v_{2} q c_{2}} \frac{\left(1 /\left(q c_{1}\right)\right) x f\left(x /\left(q c_{1}\right)\right)}{F\left(v_{2} c_{2} / c_{1}\right)} d x\right) .
\end{aligned}
$$

which can be solved numerically, similarly to the bid-based case in the previous subsection.

Example 3: Assume that $F$ is the cdf of a valuation uniformly distributed over $[0, a]$. The last two equations of the above system become

$$
\begin{aligned}
q c_{1} \mathbb{E}\left[R_{1} \mid v_{1}\right] & =\left(1-p_{2}\right) r_{r}+p_{2}\left(\frac{r_{r}^{2}}{2 q v_{1} c_{1}}+\frac{q v_{1} c_{1}}{2}\right) \\
q c_{2} \mathbb{E}\left[R_{2} \mid v_{2}\right] & =\left(1-p_{1}\right) r_{r}+p_{1}\left(\frac{r_{r}^{2}}{2 q v_{2} c_{2}}+\frac{q v_{2} c_{2}}{2}\right) .
\end{aligned}
$$

This again expresses $p_{2}$ in terms of $p_{1}$ (and reciprocally $p_{1}$ in terms of $p_{2}$ ), which can be solved numerically. For instance, with $\lambda=100, a=20, q=0.5, b_{1}=10, c_{1}=0.5, b_{2}=40$, $c_{2}=0.4, v_{1}=8, v_{2}=5$ and $r_{r}=0.125$, taking care of the four cases $\left(p_{1}<1\right.$ and $\left.p_{2}<1\right),\left(p_{1}=1\right.$ and $\left.p_{2}<1\right)$, $\left(p_{1}<1\right.$ and $\left.p_{2}=1\right)$, and $\left(p_{1}=1\right.$ and $\left.p_{2}=1\right)$, we get a single solution,

$$
p_{1}=0.1992217899 \text { and } p_{2}=1 .
$$

\section{Game Between adVertisers}

The bidding and winning probabilities, and expected revenues being computed as described above, the advertisers have to decide which proportion of their budget they will allocate to a given SE.

We consider in this section two SEs and two advertisers. Remark that the winning probabilities and revenues of advertiser $i$ at each SE depend not only on her strategy (the proportion $\beta_{i}$ allocated to SE 1 and $1-\beta_{i}$ allocated to SE 2 ) but also on the strategy of the competitor. The natural modeling framework is therefore that of non-cooperative game theory, and the equilibrium notion that of a Nash equilibrium. We recall that a Nash equilibrium is a profile of proportion strategies $\left(\beta_{1}^{*}, \beta_{2}^{*}\right)$ such that no advertiser can increase her revenue by unilaterally changing her strategy, i.e., a point $\left(\beta_{1}^{*}, \beta_{2}^{*}\right)$ such that $\forall \beta_{1}, \beta_{2} \in[0,1]$,

$$
U_{1}\left(\beta_{1}^{*}, \beta_{2}^{*}\right) \geq U_{1}\left(\beta_{1}, \beta_{2}^{*}\right) \text { and } U_{2}\left(\beta_{1}^{*}, \beta_{2}^{*}\right) \geq U_{2}\left(\beta_{1}^{*}, \beta_{2}\right) \text {. }
$$




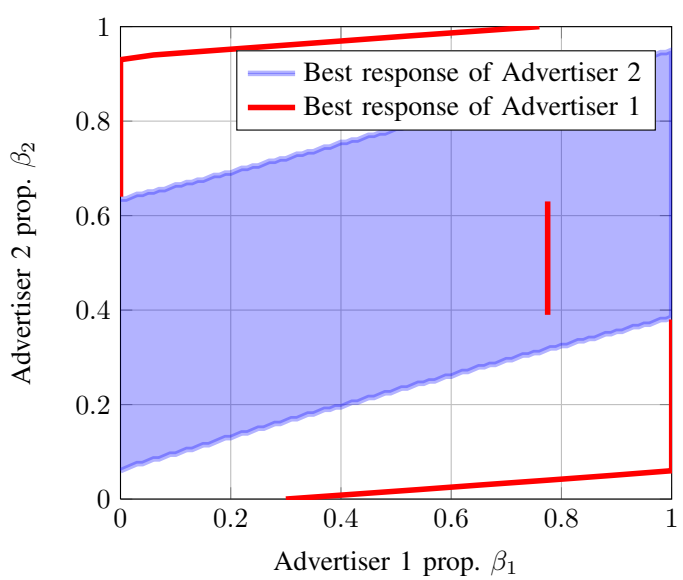

Figure 1. Best response curves when bid-based pricing is implemented at both engines.

To determine the Nash equilibria (if any) of the game in practice, we define the best response of each advertiser as a function of the strategy of its opponent. Those best response functions are

$$
\begin{aligned}
& \mathrm{BR}_{1}\left(\beta_{2}\right)=\arg \max _{\beta \in[0,1]} U_{1}\left(\beta, \beta_{2}\right) \text { and } \\
& \operatorname{BR}_{2}\left(\beta_{1}\right)=\arg \max _{\beta \in[0,1]} U_{2}\left(\beta_{1}, \beta\right) .
\end{aligned}
$$

A Nash equilibrium is simply a point $\left(\beta_{1}^{*}, \beta_{2}^{*}\right)$ for which $\operatorname{BR}_{1}\left(\beta_{2}^{*}\right)=\beta_{1}^{*}$ and $\mathrm{BR}_{2}\left(\beta_{1}^{*}\right)=\beta_{2}^{*}$. Graphically, if we draw the two best response curves on the same figure, the set of Nash equilibria is then the (possibly empty) set of intersection points of those curves.

As an illustration, Figure 1 displays the best response curves when both SEs implement bid-based pricing and with parameters $a=20$ (still choosing a uniform distribution for valuations), $\alpha=0.6, \lambda=100, q_{1}=0.5, q_{2}=0.6$ (hence a different CTR at the SEs), $b_{1}=5, c_{1}=0.5, b_{2}=20, c_{2}=0.4$, $v_{1}=10, v_{2}=9$ and $p_{r}=0.1$ at both SEs. It can be seen that for each fixed $\beta_{1}$, there is actually an interval for the best response of Advertiser 2, and that interval is of constant size, with bounds increasing linearly with $\beta_{1}$. This gives the domain in blue. For the best response of Advertiser 1 in terms of $\beta_{2}$, we obtain the red curve. The only values when the blue domain and the red curve intersect are $\{0.775\} \times[0.39,0.63]$, giving the set of Nash equilibria.

To better understand what happens, we draw in Figures 2 and 3 for fixed but arbitrary values $\beta_{i}$ of the opponent $i$ (respectively $\beta_{2}=0.95$ and $\beta_{1}=0.21$ ) the revenues of advertisers in terms of their strategy. The maximum total revenue will give the best response of the advertiser. We can observe on Figure 2 that for $\beta_{2}=0.95$ there is a (single) maximum at $\beta_{1} \approx 0.175$, which is in accordance with the red best-response curve of Figure 1. Figure 3 shows that the maximum revenue of Advertiser 2 is obtained for an interval, again in accordance with the (blue) best-response domain of Figure 1. The constant value of revenues is actually due to the fact that the probability of bidding is then maximal, equal to

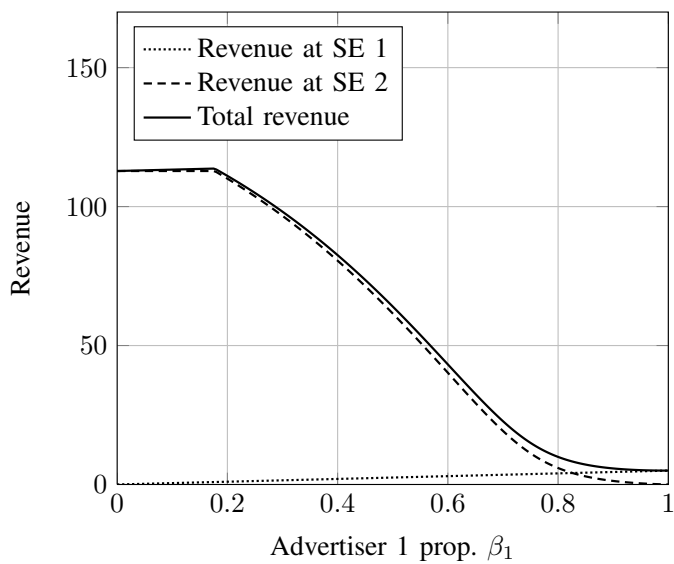

Figure 2. Revenues of Advertiser 1 in terms of $\beta_{1}$.

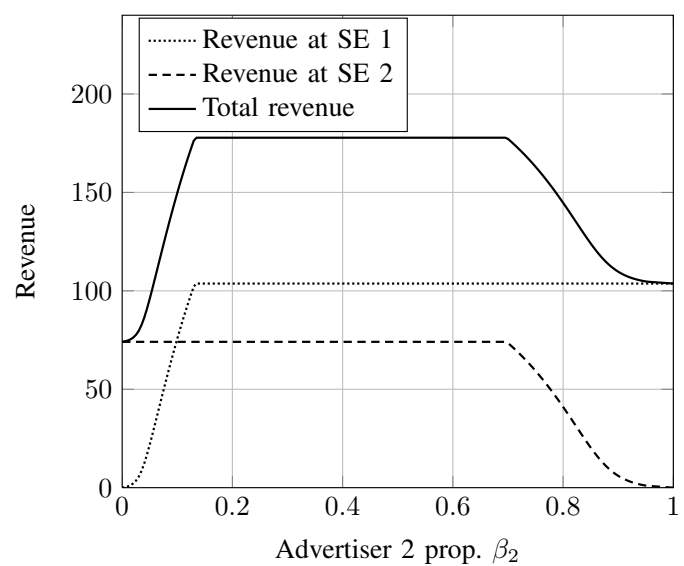

Figure 3. Revenues of Advertiser 2 in terms of $\beta_{2}$.

1 , and therefore independent of the submitted budget; in other words, the budget is not fully spent. In order to illustrate that, we display the winning probabilities of advertisers in terms of the budget proportion allocated to $\operatorname{SE} 1$, when the $\beta_{i}$ of the competitor is fixed to the same value than in Figures 2 and 3. Figure 4 draws the winning probabilities in terms of $\beta_{1}$ when $\beta_{2}=0.95$. There is no interval where $p_{1}$ and $p_{2}$ are constant at the same SE, resulting in no constant revenue. On the other hand, this is not true anymore in Figure 5 where bidding probabilities are expressed in terms of $\beta_{2}$ when $\beta_{1}=0.21$. Remark also that the intervals correspond with those of constant revenues in Figure 3.

Similarly to the case where both SEs implement bid-based ranking (noted B-B case), we can investigate the advertisers' behavior when all other combinations are adopted:

- bid-based for SE 1 and revenue-based for SE 2 (noted B-R case),

- revenue-based for SE 1 and bid-based for SE 2 (noted R-B case),

- revenue-based for both SEs (noted R-R case).

Figures 6, 7 and 8 display the best-response curves for those three cases. When revenue-based ranking is implemented, the reserve revenue $r_{r}=0.025$ is used, which corresponds 


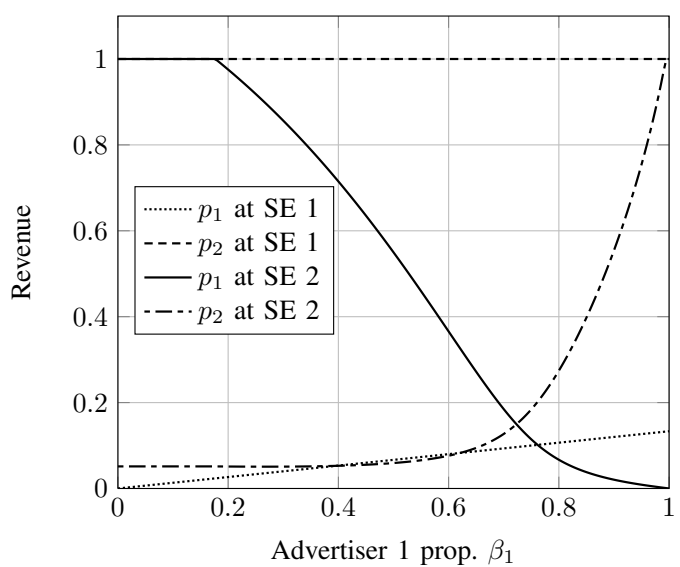

Figure 4. Bidding probabilities in terms of $\beta_{1}$ (with $\beta_{2}=0.95$ ).

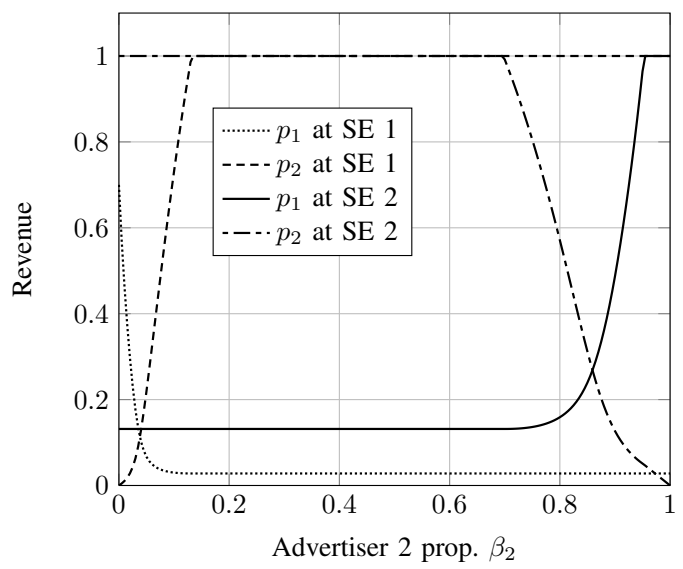

Figure 5. Bidding probabilities in terms of $\beta_{2}$ (with $\beta_{1}=0.21$ ).

approximately to the quantity $q c p_{r}$ when bid-based ranking is used.

As can be checked on the figures, we end up with the following sets of Nash equilibria:

- For the B-B case, all the profiles $\left(\beta_{1}, \beta_{2}\right) \in\{0.775\} \times$ $[0.39,0.63]$

- for the B-R case, it is $\{0\} \times[0.39,0.695]$;

- for the R-B case, $\{0.97\} \times[0.34,0.63]$;

- for the R-R case, $\{0\} \times[0.34,0.695]$.

It can be noted that, with our set of parameters, when SE 2 implements revenue-based ranking, Advertiser 1's strategy at a Nash equilibrium is to put all her budget on SE 2. Remark also that even if there are multiple equilibria, the revenues of advertisers are unique. Indeed, those equilibria correspond to the same bidding strategies, where advertiser 2 bids with probability 1 on both SEs (every budget repartition such that advertiser 2 cannot exceed the pre-affected budget on any SE leads to that same bidding behavior).

\section{WHICH MECHANISM TO IMPLEMENT AT THE SE LEVEL?}

The behavior of advertisers being known for all combinations of ranking strategies, the SEs can wonder what strategy to implement beforehand, anticipating the advertisers'

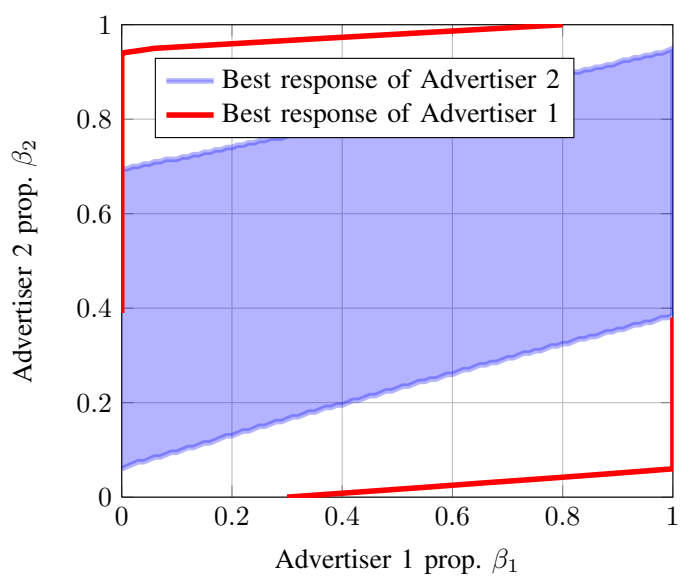

Figure 6. Best response curves when bid-based pricing is implemented at SE 1 and revenue-based at SE 2.

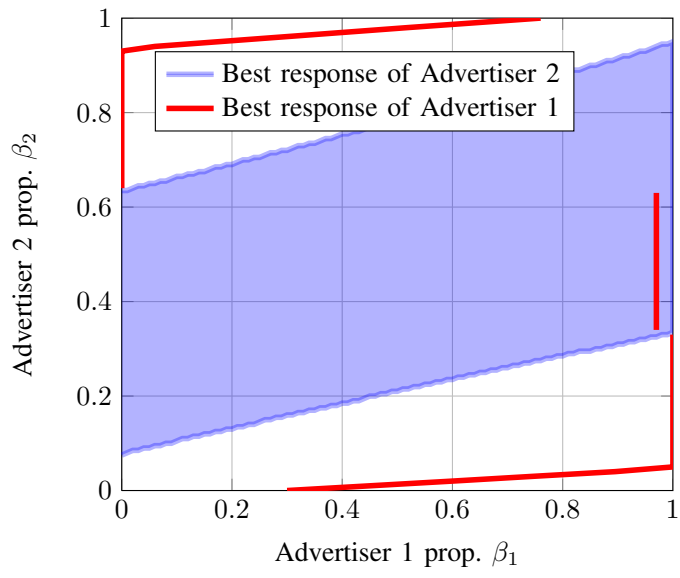

Figure 7. Best response curves when bid-based pricing is implemented at SE 2 and revenue-based at SE 1.

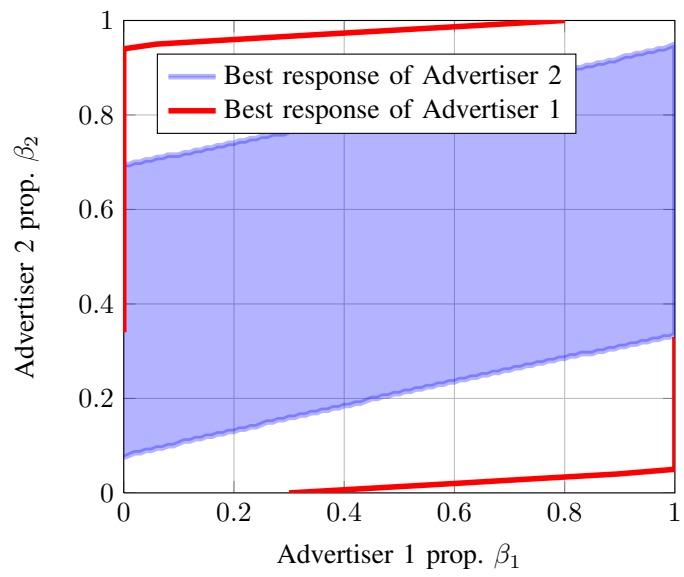

Figure 8. Best response curves when revenue-based pricing is implemented at both engines. 
decisions. SE 1 and 2 seek to maximize their revenues from advertisement, being respectively

$$
\begin{aligned}
\operatorname{Rev}_{1}= & \alpha \lambda p_{1} q_{1} c_{1} \mathbb{E}\left[R_{1} \mid v_{1}\right] w_{1}\left(v_{1}\right)+\alpha \lambda p_{2} q_{1} c_{2} \mathbb{E}\left[R_{2} \mid v_{2}\right] w_{2}\left(v_{2}\right) \\
\operatorname{Rev}_{2}= & (1-\alpha) \lambda \tilde{p}_{1} q_{2} c_{1} \mathbb{E}\left[\tilde{R}_{1} \mid v_{1}\right] \tilde{w}_{1}\left(v_{1}\right) \\
& +(1-\alpha) \lambda \tilde{p}_{2} q_{2} c_{2} \mathbb{E}\left[\tilde{R}_{2} \mid v_{2}\right] \tilde{w}_{2}\left(v_{2}\right),
\end{aligned}
$$

where the tilde sign distinguishes values specific to SE 2 (e.g., $p_{1}$ is the bidding probability of provider 1 at SE 1 while $\tilde{p}_{1}$ denotes her bidding probability at SE 2).

Here again, we have a non-cooperative game, and the equilibrium situation is that of Nash equilibrium. It is here a revenue profile $\left(R_{1}, R_{2}\right)$ such that no engine can unilaterally increase her revenue. The SE revenue values are provided in Table I, described here in the case of the numerical values obtained in Section IV, but it can be derived similarly for other values.

Table I

GAME ON RANKING STRATEGIES AMONG SES. EACH ELEMENT GIVES $\left(\mathrm{REV}_{1}, \mathrm{REV}_{2}\right)$ IN TERMS OF THE RULES USED BY SE 1 (LINE) AND SE2 (COLUMN)

\begin{tabular}{|c|cc|}
\hline & $\mathrm{B}$ & $\mathrm{R}$ \\
\hline $\mathrm{B}$ & $(10.15,3.62)$ & $(1.20,11.06)$ \\
$\mathrm{R}$ & $(11.32,1.32)$ & $(1.50,11.06)$ \\
\hline
\end{tabular}

One can note that the best strategy for SE 2 is to always play the revenue-based strategy. Actually, it can be readily checked that the combination of strategies R-R is a Nash equilibrium. On the other hand, we have found other sets of parameters such that $\mathrm{B}-\mathrm{R}$ is an equilibrium. This kind of game has therefore to be closely looked at by search engines.

\section{CONCLUSIONS}

We have defined a model describing two search engines in competition for advertisers in adword auctions. We have derived, in the case where each engine proposes a single advertisement slot and advertisers have a budget per unit of time that they want to optimally split among engines, how advertisers should behave depending on the ranking strategies of engines. On top of this advertisers' game, we have illustrated how engines can (competitively) play on the ranking strategy.

As directions for future research, we would like to extend our study to the situation where SEs propose more than one slot. We would also like to study more specifically the case of Google against Yahoo!, the two main engines, to better understand Yahoo!'s move to follow Google with revenuebased ranking. This requires to carefully compute the system parameters and distributions.

\section{ACKNOWLEDGMENT}

This work was supported by Euro-NF Network of Excellence (http://euronf.enst.fr/) and the French research agency through the CAPTURES project (http://captures.inria.fr/).

\section{REFERENCES}

[1] H. Varian, "Position auctions," International Journal of Industrial Organization, vol. 25, pp. 1163-1178, 2005.

[2] D. Liu, J. Chen, and A. Whinston, "Competing keyword auctions," in Proc. of 4th Workshop on Ad Auctions, Chicago, IL, USA, Jul 2008.

[3] W. Vickrey, "Counterspeculation, auctions, and competitive sealed tenders," Journal of Finance, vol. 16, no. 1, pp. 8-37, Mar 1961.

[4] T. Groves, "Incentives in teams," Econometrica, vol. 41, no. 3, pp. 617631, Jul 1973.

[5] B. Edelman, M. Ostrovsky, and M. Schwarz, "Internet Advertising and the Generalized Second-Price Auction: Selling Billions of Dollars Worth of Keywords," The American Economic Review, vol. 97, no. 1, pp. 242 259, 2007.

[6] P. Jordan and M. Wellman, "Designing an ad auctions game for the trading agent competition," in IJCAI-09 Workshop on Trading Agent Design and Analysis (TADA), Pasadena, California, USA, Jul 2009.

[7] S. Lahaie, D. Pennock, A. Saberi, and R. Vohra, "Sponsored search auctions," in Algorithmic Game Theory, N. Nisan, T. Roughgarden, E. Tardos, and V. Vazirani, Eds. Cambridge University Press, 2007, ch. 28, pp. 699-716.

[8] P. Maillé, E. Markakis, M. Naldi, G. Stamoulis, and B. Tuffin, "An overview of research on sponsored search auctions," 2010, available at http://www.irisa.fr/dionysos/pages_perso/tuffin/Publis/Survey-v4.pdf.

[9] R. Gonen, "Characterizing optimal syndicated sponsored search market design," in Proc. of the 5th Workshop on Ad Auctions, Stanford, CA, USA, Jul 2009.

[10] I. Ashlagi, D. Monderer, and M. Tennenholtz, "Competing ad auctions," in Proc. of 4th Workshop on Ad Auctions, Chicago, IL, USA, Jul 2008.

[11] J. Liu and D. Chiu, "Mathematical modeling of competition in sponsored search market," in Proc. of NetEcon, 2010.

[12] M. Osborne and A. Rubenstein, A Course on Game Theory. MIT Press, 1994.

[13] P. Maillé and B. Tuffin, "On the ranking strategy in adword auctions," in Proc. of the 8th International Conference on Information Technology: New Generations (ITNG), Las Vegas, USA, April 2011. 